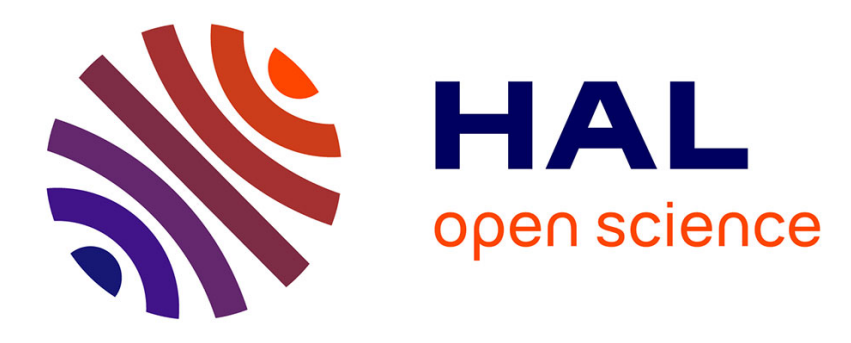

\title{
Humanoid walking with compliant soles using a deformation estimator
}

Giovanni de Magistris, Adrien Pajon, Sylvain Miossec, Abderrahmane

Kheddar

\section{- To cite this version:}

Giovanni de Magistris, Adrien Pajon, Sylvain Miossec, Abderrahmane Kheddar. Humanoid walking with compliant soles using a deformation estimator. ICRA: International Conference on Robotics and Automation, May 2016, Stockholm, Sweden. pp.1757-1762, 10.1109/ICRA.2016.7487319 . hal01278856

\section{HAL Id: hal-01278856 \\ https://hal.science/hal-01278856}

Submitted on 25 Feb 2016

HAL is a multi-disciplinary open access archive for the deposit and dissemination of scientific research documents, whether they are published or not. The documents may come from teaching and research institutions in France or abroad, or from public or private research centers.
L'archive ouverte pluridisciplinaire HAL, est destinée au dépôt et à la diffusion de documents scientifiques de niveau recherche, publiés ou non, émanant des établissements d'enseignement et de recherche français ou étrangers, des laboratoires publics ou privés. 


\title{
Humanoid walking with compliant soles using a deformation estimator
}

\author{
Giovanni De Magistris ${ }^{1,3}$, Adrien Pajon ${ }^{1,3}$, Sylvain Miossec ${ }^{2}$ and Abderrahmane Kheddar ${ }^{1,3}$
}

\begin{abstract}
We study the effect of soft (i.e. compliant) soles in humanoid walking. Adding compliance at the foot sole is important for shock absorption during touchdown and to better cast the ground roughness. But, subsequent deformation needs to be considered in the attitude stabilization. We propose a sole deformation estimator used in the controller design, to ensure that the desired ZMP for stability requirement is fulfilled. We performed two comparison experiments on the HRP4 humanoid robot with/without the estimator. Experiments showed how the estimator enhances the ZMP stabilization during walking and prevents the robot from falling down.
\end{abstract}

\section{INTRODUCTION}

Humanoids robots move by alternating phases of contact creations and removals [1]. The links of the robot being rigid and without any shock absorbing mean, reducing impacts can only be made through contact transitions with nearly zero speed. This would constraint a lot dynamic walking. Therefore, compliant mechanisms are commonly added in humanoid robots. Their main role is to absorb shocks at impacts and prohibit their propagation along the entire linkage. The most common approach is to add a compliant mechanism at the ankle [2], [3], so that the force sensor is also protected. Unfortunately, such a compliant mechanism also acts as a passive joint which deformation is hardly measurable. This makes the robot attitude difficult to control, especially in complex maneuvers [4]. Another compliance is attached to the bottom part of the foot [5]. In fact, it is most common that humanoid robots have a thin rubber sole attached to the bottom part of the robot foot. Because it is thin, large part of the landing shocks are rather absorbed at the ankle-embedded compliance.

In this paper, we investigate the effect of removing ankle flexibilities and adding thick soft soles instead (see Fig. 1). By doing this, heel strike impact is absorbed while improving stiffness in flat foot position by a better contact-surface casting. But such a compliant sole has a varying compliance due to change of the contact area. In order to improve balance of the robot during the walk and to cope with such varying compliance, we devised a deformation estimator and we assessed it with robot experiments.

\section{Problem formulation}

Model-based planning and control does not take into account flexibilities (at the ankle or at the sole); thus generated trajectories do not guarantee balance viability. The control

This work is supported in part by the FP7 KOROIBOT.

${ }^{1}$ CNRS-AIST Joint Robotics Laboratory (JRL), UMI3218/RL, Japan.

2 PRISME Laboratory, University of Orléans, France

3 CNRS-UM, LIRMM Interactive Digital Human, France

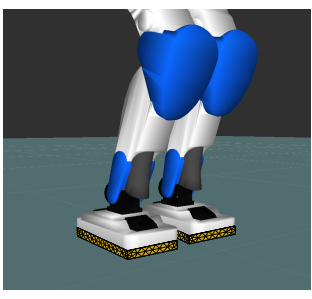

(a) (b)

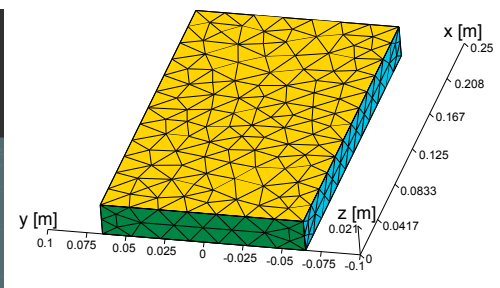

Fig. 1: (a): Rectangular parallelepiped soles mounted on HRP-4's feet; (b): meshed sole with 1494 tetrahedron elements

has to compensate both for the errors in orientation/position due to flexibility deformation and other uncertainties.

The algorithm developed in this paper is able to well estimate the deformation using the mechanical properties of the flexible sole (for instance, finite element model (FEM) and mechanical laws of contact) to enhance the robot stabilizer to maintain the balance during walking. The ankle reference trajectories change according to sole deformations.

The control scheme is illustrated in Fig. 2.

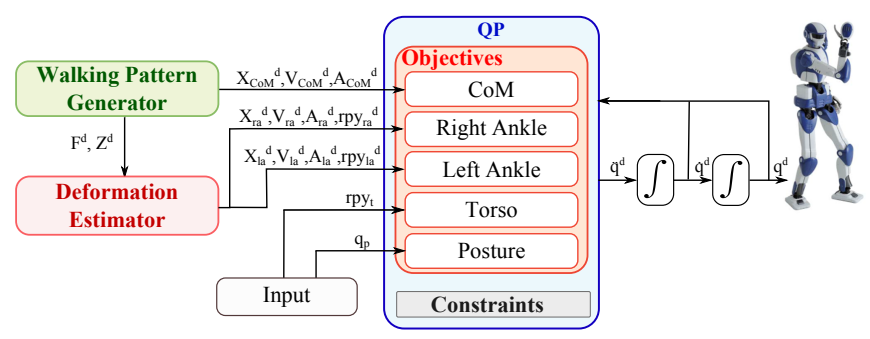

Fig. 2: Control scheme

The walking pattern generator (WPG) in Fig. 2 finds smooth fifth-order polynomial ZMP trajectory of each foot in contact with the ground during single support phase (SSP) and double support phases (DSP). To date, no existing WPG fulfills such requirements (especially the study of ZMP trajectory and force in DSP). In this work, the walking control formulation takes both single-support and double-support phases into consideration as a quadratic programming $(\mathrm{QP})$ optimization problem.

Using the simple pendulum model proposed in [6], we simplified the expression of the energy consumption in [7] 
into:

$$
\begin{aligned}
c= & \int_{t_{i}}^{t_{f}} \lambda\left\|\boldsymbol{F}_{\text {сом }}(t)\right\|^{2}+(1-\lambda)\left\|\boldsymbol{\Gamma}_{a}(t)\right\|^{2} \mathrm{dt} \\
& +\epsilon \int_{t_{i}}^{t_{f}}\left(\left\|\boldsymbol{A}_{1}(t)\right\|^{2}+\left\|\boldsymbol{A}_{2}(t)\right\|^{2}\right) \mathrm{dt}
\end{aligned}
$$

where $\lambda, \epsilon \in \mathbb{R}_{+}$are the weights of each criterion; subscript 1 denotes the foot that leaves the floor at the end of DSP, and subscript 2 the foot that comes in contact at the beginning of DSP; $\boldsymbol{F}_{\text {сом }}$ denotes the center-of-mass (COM) force; $\boldsymbol{\Gamma}_{a}$ is the torque at ankle joint $a$.

Compared to [8], we added a third criterion to the cost function (1) to obtain a heel-toe human like ZMP trajectory under each foot. $\boldsymbol{A}_{1}$ and $\boldsymbol{A}_{2}$ are respectively the acceleration of $\mathrm{ZMP}_{1}$ and $\mathrm{ZMP}_{2}$ in DSP. Therefore the cost function term dependent of these two terms allows to generate $\mathrm{ZMP}_{1}$ and $\mathrm{ZMP}_{2}$ trajectories in the walking direction and to minimize foot angular acceleration. The acceleration of global ZMP in SSP is taken into account in the criterion dependent on the COM force.

The details of how to obtain ZMP and COM trajectories and how to formulate the QP optimization problem are given in [8].

We divided the sole shape $\Omega$ in 1494 tetrahedron elements (see meshed sole in Fig. 1) and the surface describing the volume $\partial \Omega$ into two non-empty disjoint parts:

$$
\partial \Omega=S_{D} \cup S_{S}, \quad \text { with } \quad S_{D} \cap S_{S}=\emptyset
$$

$S_{D}$ is the surface attached to the lower part of the robot foot (Dirichlet surface) and $S_{S}$ is the surface that could be in contact with the ground.

We also called $\Omega_{I}$ the interior volume of $\Omega$.

To model the mechanical properties of the sole, we used a static linear FEM. This model is valid when the node displacements relative to the object size is relatively low (small deformations [9]) and the system response is fast enough to neglect the dynamic phenomena compared to $\boldsymbol{F}^{\mathrm{d}}$ and $Z^{\mathrm{d}}$ variations.

Using a static linear FEM, the displacements at each point of the mesh depend on external forces applied to the same points and we can then write the following relationship:

$$
\boldsymbol{K}^{\underline{U} \boldsymbol{U}}={ }^{l} \underline{\boldsymbol{F}}
$$

where ${ }^{l} \underline{\boldsymbol{F}} \in \mathbb{R}^{N}$ is the vector of generalized forces (nodal forces) and $\underline{U} \in \mathbb{R}^{N}$ is the vector of the node displacements. The superscript $l$ before a letter denotes a vector expressed in the sole frame and no superscript means that the vector is expressed in the world frame.

The displacements of the Dirichlet nodes are known and for this reason, we can write the system (3) as:

$$
\left[\begin{array}{lll}
\boldsymbol{K}_{D D} & \boldsymbol{K}_{D I} & \boldsymbol{K}_{D S} \\
\boldsymbol{K}_{I D} & \boldsymbol{K}_{I I} & \boldsymbol{K}_{I S} \\
\boldsymbol{K}_{S D} & \boldsymbol{K}_{S I} & \boldsymbol{K}_{S S}
\end{array}\right]\left[\begin{array}{l}
\underline{\mathbf{0}} \\
{ }^{l} \underline{\boldsymbol{U}}_{I} \\
{ }^{\boldsymbol{U}_{S}}
\end{array}\right]=\left[\begin{array}{l}
{ }^{l} \boldsymbol{F}_{D} \\
{ }^{l} \boldsymbol{F}_{I} \\
{ }^{l} \boldsymbol{F}_{S}
\end{array}\right]
$$

where $I, D$ and $S$ denotes respectively nodes of $\Omega_{I}, S_{D}$ and $S_{S}$.

As in [10], we considered that external forces can be only applied on the surface $S_{D}$ and thus the internal forces $\underline{\boldsymbol{F}_{I}}=$ $\underline{\text { 0. }}$ Therefore the system becomes:

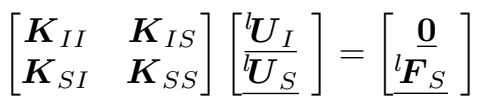

From (5), we can write a condensed linear elasticity law [10]:

$$
\begin{aligned}
\underline{\boldsymbol{U}_{S}} & =\left(\boldsymbol{K}_{S S}-\boldsymbol{K}_{S I} \boldsymbol{K}_{I I}^{-1} \boldsymbol{K}_{I S}\right)^{-1}{ }^{l} \boldsymbol{F}_{S} \\
& =\boldsymbol{K}_{S}^{-1}{ }^{l} \boldsymbol{F}_{S}=\boldsymbol{C}_{S}{ }^{l} \boldsymbol{F}_{S} \\
\underline{\boldsymbol{U}_{I}} & =-\boldsymbol{K}_{I I}^{-1} \boldsymbol{K}_{I S} \underline{\boldsymbol{U}_{S}}
\end{aligned}
$$

where $\boldsymbol{K}_{S}$ is the stiffness surface matrix and $\boldsymbol{C}_{S}$ is the compliance surface matrix.

To find the deformation of each sole node, we first calculated the displacement ${ }^{l} U_{S}$ induced by the contact with the ground, and then calculated the displacement ${ }^{U_{I}}$ using the second equation in (6).

\section{DEFORMATION ESTIMATOR}

In this section we explain our new algorithm that finds the ankle position and orientation at each instant of time $q$ taking into account the sole deformation. The deformation is calculated using the desired interaction force $\boldsymbol{F}^{\mathrm{d}}(t)$ and ZMP trajectory $\boldsymbol{Z}^{\mathrm{d}}(t)$ given by the WPG (see control scheme 2 ). This algorithm is illustrated in Fig. 4 and it is composed by two main blocks: frictional contact problem (see section IIIA) and update sole position and orientation (see section IIIB).

\section{A. Frictional contact problem}

Supposing that we discretize the simulation in $r$ time steps, the method explained below finds the contact space displacement vector $\underline{\boldsymbol{\delta}}$ and contact force vector $\underline{\boldsymbol{F}}$ respecting Signorini's and Coulomb's laws given a sole position and orientation at an instant of time $q$.

The inputs of this algorithm are:

1) ${ }^{l} \underline{P}^{\text {free }}:$ node positions

2) $\bar{C}_{S}$ : surface compliance matrix calculated using the FEM model in equation (6)

3) ${ }^{l} \underline{\boldsymbol{P}}^{q-1}$ : node positions at the previous time step

4) $\boldsymbol{O}_{l}$ : foot position vector

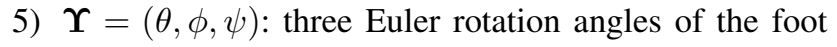
rotation

The outputs of this algorithm are:

1) $\underline{\delta}$ : relative position between the sole node positions and the ground position (see Fig. 3). It is called node contact space displacement vector

2) $\underline{F}$ : node contact force vector

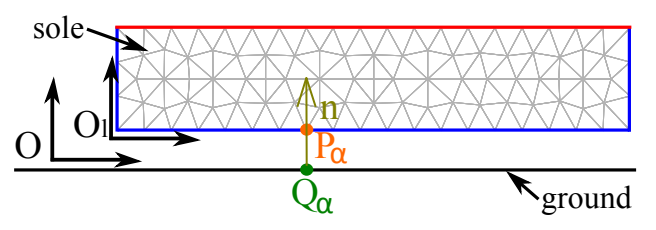

Fig. 3: Contact between the sole and the ground 
For a contact point $\alpha, \boldsymbol{\delta}_{\alpha}$ is defined by:

$$
\boldsymbol{\delta}_{\alpha}=\boldsymbol{P}_{\alpha}-\boldsymbol{Q}_{\alpha}
$$

where $Q_{\alpha}$ is a reference point on the ground.

Using equation (7), we can define the Signorini's law to assure the non-interpenetration between the sole and ground and the Coulomb's law characterizes the dry friction as:

1) Signorini's law:

$$
\mathbf{0} \leq \boldsymbol{F}_{n, \alpha} \perp \boldsymbol{\delta}_{n, \alpha} \geq \mathbf{0}
$$

where $\boldsymbol{F}_{\alpha}$ is the contact force applied to a contact node $\alpha$. In this paper, we denote with the subscript $n$ the normal component of the vector $\boldsymbol{v}_{n}=\mathbf{n} \boldsymbol{v}$ where $\mathbf{n}=$ $\left[\begin{array}{lll}0 & 0 & 1\end{array}\right]$ is the normal vector and with the subscript $t$ the tangential components of the vector $\boldsymbol{v}_{t}=\mathbf{t} \boldsymbol{v}$, where $\mathbf{t}=\left[\begin{array}{lll}1 & 0 & 0 \\ 0 & 1 & 0\end{array}\right]$.

2) Coulomb's law. We can distinguish two complementarity conditions for stick and slip motions:

a) Stick condition:

$$
\left\|\boldsymbol{F}_{t, \alpha}\right\|<\mu\left|F_{n, \alpha}\right|, \quad \boldsymbol{\delta}_{t, \alpha}=0
$$

\section{b) Slip condition:}

$$
\boldsymbol{F}_{t, \alpha}=-\mu F_{n, \alpha} \frac{\boldsymbol{\delta}_{t, \alpha}}{\left\|\boldsymbol{\delta}_{t, \alpha}\right\|}
$$

where $\mu$ is the friction coefficient.

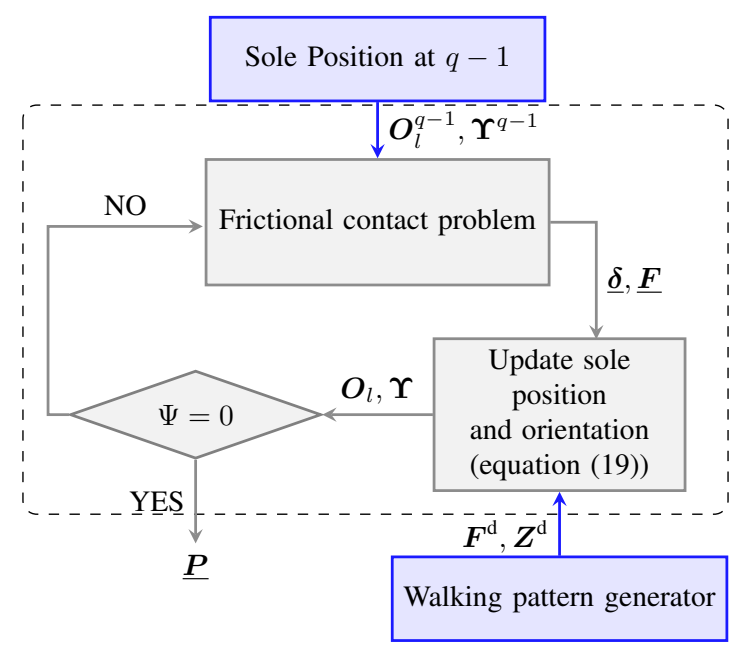

Fig. 4: Framework for contact handling to obtain $\boldsymbol{F}^{\mathrm{d}}$ and $\boldsymbol{Z}^{\mathrm{d}} \cdot q-1$ is the previous time step and $\Psi=$ $\left[\boldsymbol{F}^{\mathrm{tot}}-\boldsymbol{F}^{\mathrm{d}}, \boldsymbol{Z}-\boldsymbol{Z}^{\mathrm{d}}, \boldsymbol{\Gamma}_{Z^{\mathrm{d}}, n}\right]^{T}$

To solve this problem, we define $\boldsymbol{P}_{\alpha}$ and $\boldsymbol{Q}_{\alpha}$ of equation (7). To do that, we use the displacement $\underline{\boldsymbol{U}_{S}}$ of equation (6) (in absolute frame):

$$
\underline{\boldsymbol{U}_{S}}=\underline{\boldsymbol{P}}-\underline{\boldsymbol{P}}^{\mathrm{free}}
$$

and then, we define the sole surface node positions as:

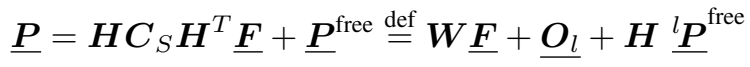

$\boldsymbol{W}$ is the Delassus operator [11]; $\boldsymbol{H}=\operatorname{diag}(\boldsymbol{R}, \ldots, \boldsymbol{R})$, where $\boldsymbol{R}$ is the foot orientation matrix defined by $\boldsymbol{\Upsilon}=$ $(\theta, \phi, \psi)$. The superscript $T$ denotes the transpose operator.

Supposing the absolute frame on the flat floor, and hence $\mathbf{n} \boldsymbol{Q}_{\alpha}=0$, the normal component of the displacement vector (7) for each contact point $\alpha$ is:

$$
\begin{aligned}
\boldsymbol{\delta}_{n, \alpha} & =\mathbf{n} \boldsymbol{O}_{l}+\mathbf{n} \boldsymbol{R}^{l} \boldsymbol{P}_{\alpha}^{\text {free }}+\mathbf{n} \sum_{\beta=1}^{m}\left(\boldsymbol{W}_{\alpha \beta} \boldsymbol{F}_{\beta}\right) \\
& \stackrel{\text { def }}{=} \boldsymbol{\delta}_{n, \alpha}^{\text {free }}+\mathbf{n} \sum_{\beta=1}^{m}\left(\boldsymbol{W}_{\alpha \beta} \boldsymbol{F}_{\beta}\right)
\end{aligned}
$$

where $\boldsymbol{W}_{\alpha \beta} \stackrel{\text { def }}{=}\left[\begin{array}{c}W_{t} \\ W_{n}\end{array}\right] \stackrel{\text { def }}{=}\left[\begin{array}{cc}W_{t t} & W_{t n} \\ W_{n t} & W_{n n}\end{array}\right]$ is the $3 \times 3$ Delassus' operator coupling the contacts $\alpha$ and $\beta$.

The tangential component of the contact space displacement (7) is:

$$
\begin{aligned}
\boldsymbol{\delta}_{t, \alpha} & =\mathbf{t} \boldsymbol{O}_{l, \alpha}+\mathbf{t} \boldsymbol{R}^{l} \boldsymbol{P}_{\alpha}^{\mathrm{free}}+\mathbf{t} \sum_{\beta=1}^{m}\left(\boldsymbol{W}_{\alpha \beta} \boldsymbol{F}_{\beta}\right)-\mathbf{t} \boldsymbol{Q}_{\alpha} \\
& \approx \mathbf{t} \boldsymbol{O}_{l, \alpha}+\mathbf{t} \boldsymbol{R}^{l} \boldsymbol{P}_{\alpha}^{\mathrm{free}}+\mathbf{t} \sum_{\beta=1}^{m}\left(\boldsymbol{W}_{\alpha \beta} \boldsymbol{F}_{\beta}\right)-\mathbf{t} \boldsymbol{P}_{\alpha}^{(q-1)} \\
& \stackrel{\text { def }}{=} \boldsymbol{\delta}_{t, \alpha}^{\mathrm{free}}+\mathbf{t} \sum_{\beta=1}^{m}\left(\boldsymbol{W}_{\alpha \beta} \boldsymbol{F}_{\beta}\right)
\end{aligned}
$$

In case of a node already in contact at $q-1$, the contact reference point is the previous node position $\boldsymbol{P}_{\alpha}^{(q-1)}$. In case of a new contact, we project on the ground the node position $\boldsymbol{P}_{\alpha}^{(q-1)}$ as an approximation of the reference contact position. In fact, real contact takes place at an intermediate time between previous and current time step, and a node may have moved a little in the tangential direction. Precise contact position could be found, but at the price of a variable step size and event driven simulation, which is computationally costly when many contacts occur. Additionally, this approximation is good enough when the time-step size is sufficiently small.

From (13) and (14), we obtain the contact space displacement for a contact node $\alpha$ :

$$
\begin{aligned}
\boldsymbol{\delta}_{\alpha} & =\boldsymbol{O}_{l}+\boldsymbol{R}^{l} \boldsymbol{P}_{\alpha}^{\mathrm{free}}-\left[\begin{array}{c}
\boldsymbol{P}_{t, \alpha}^{(q-1)} \\
0
\end{array}\right]+\sum_{\beta=1}^{m} \boldsymbol{W}_{\alpha \beta} \boldsymbol{F}_{\beta} \\
& \stackrel{\text { def }}{=} \boldsymbol{\delta}_{\alpha}^{\mathrm{free}}+\sum_{\beta=1}^{m} \boldsymbol{W}_{\alpha \beta} \boldsymbol{F}_{\beta}
\end{aligned}
$$

Then, the system that we need to solve for a frictional contact problem is:

$$
\underline{\boldsymbol{\delta}}=\underline{\boldsymbol{O}}_{l}+\boldsymbol{H}^{l} \underline{\boldsymbol{P}}^{\text {free }}-\underline{\boldsymbol{Q}}+\boldsymbol{W} \underline{\boldsymbol{F}}
$$

where $\underline{\boldsymbol{Q}}=\left[\left[\begin{array}{c}\boldsymbol{P}_{t, 1}^{(q-1)} \\ 0\end{array}\right]^{T} \ldots\left[\begin{array}{c}\boldsymbol{P}_{t, m}^{(q-1)} \\ 0\end{array}\right]^{T}\right]^{T}$ and $m$ is the number of the contact nodes.

In the system (16), we have $3 m$ equations with $6 m$ unknowns $\underline{\boldsymbol{\delta}}$ and $\underline{\boldsymbol{F}}$. To find the $3 m$ equations that we need to solve the system, we can distinguish two cases for each contact node $\alpha$ :

1) Stick: From (9) $\boldsymbol{\delta}_{\alpha}=0 \rightarrow$, we have $3 z$ equations. $z$ is the number of the contact in stick case

2) Slip: $\delta_{n, \alpha}=0 \rightarrow s$ equations, where $s$ is the number of the contact in slip case. Then, to solve the system 
(16), we need $2 s$ equations. To obtain this, we use the Coulomb's law (10).

For each node in the slip conditions, to respect the Coulomb's law, we use the non-linear approach in [12]. Using (15) and Coulomb's law in [12], we build for each contact $\alpha$ a system and we solve it using a Newton's method [13].

To solve the system (16), we use the iterative Gauss-Seidel method in [14], [15]. This method is very fast and applicable for real-time solution [14].

Using (8) and (13), the Signorini's problem can be solved using a LCP (Linear Complementarity Problem).

\section{B. Update sole position and orientation}

In the previous section, we detailed the frictional contact problem where the inputs are the foot position and orientation and the outputs are the contact forces and the contact space displacements.

In this section, we propose a new algorithm to calculate foot position and orientation to obtain the desired $\boldsymbol{F}^{\mathrm{d}}(t)$ and $\boldsymbol{Z}^{\mathrm{d}}(t)$ and null vertical torque at $\mathrm{ZMP} \boldsymbol{\Gamma}_{Z^{\mathrm{d}}, n}=0$ mentioned in Fig. 4.

The inputs of this algorithm are: 1) $\left.\left.{ }^{l} \underline{\boldsymbol{P}}^{\text {free }}, 2\right) \boldsymbol{C}_{S}, 3\right) \underline{\boldsymbol{P}}^{q-1}$, 4) $\left(\boldsymbol{F}^{\mathrm{d}}, \boldsymbol{Z}^{\mathrm{d}}\right)$.

The outputs of this algorithm are: 1) $\left.\boldsymbol{O}_{l}, 2\right) \Upsilon=(\theta, \phi, \psi)$, 3) $(\underline{F}, \underline{\delta})$.

Let $f$ be the function computing $\boldsymbol{F}, \boldsymbol{Z}$ and $\boldsymbol{\Gamma}_{Z^{\mathrm{d}}, n}$ from $(\underline{\boldsymbol{F}}, \underline{\boldsymbol{\delta}})$, we solve the equation

$$
f\left(\underline{\boldsymbol{F}}, \underline{\boldsymbol{\delta}}, \boldsymbol{O}_{l}, \boldsymbol{\Upsilon}\right)=\left[\begin{array}{c}
\boldsymbol{F}^{\mathrm{d}}(t) \\
\boldsymbol{Z}^{\mathrm{d}}(t) \\
0
\end{array}\right]
$$

by Newton's method.

Starting from the system (16), we have $3 m+6$ unknowns and $3 m$ equations given by the frictional contact problem explained in the previous section. The 6 remaining equations are given using the relationship between the total force $\boldsymbol{F}^{\text {tot }}$ and the nodal forces:

$$
\boldsymbol{F}^{\mathrm{tot}}=\sum_{\alpha=1}^{m} \boldsymbol{F}_{\alpha}
$$

and using the definition of ZMP and vertical resultant torque at $Z^{\mathrm{d}}$ we get:

$$
\left[\begin{array}{c}
\Gamma_{Z, t_{1}}=0 \\
\Gamma_{Z, t_{2}}=0 \\
\Gamma_{Z^{\mathrm{d}}, n}
\end{array}\right]=\sum_{\beta=1}^{m}\left[\begin{array}{c}
\left(P_{t_{2}, \beta}-Z_{t_{2}}\right) \cdot F_{n, \beta} \\
-\left(P_{t_{1}, \beta}-Z_{t_{1}}\right) \cdot F_{n, \beta} \\
\left(P_{t_{1}, \beta}-Z_{t_{1}}\right) \cdot F_{t_{2}, \beta}-\left(P_{t_{2}, \beta}-Z_{t_{2}}\right) \cdot F_{t_{1}, \beta}
\end{array}\right]
$$

where $\Gamma_{Z}$ is the resultant torque at ZMP and the subscripts $t_{1}$ and $t_{2}$ denote respectively the first and second component of the vector.

Therefore, we derive a Newton step by finding a differential relationship between $\left(\boldsymbol{F}^{\text {tot }}, \boldsymbol{Z}, \boldsymbol{\Gamma}_{Z^{\mathrm{d}}, n}\right)$ and $\left(\boldsymbol{O}_{l}, \boldsymbol{\Upsilon}\right)$ :

$$
\left[\begin{array}{l}
\boldsymbol{O}_{l}^{(g)} \\
\boldsymbol{\Upsilon}^{(g)}
\end{array}\right]=\left[\begin{array}{l}
\boldsymbol{O}_{l}^{(g-1)} \\
\boldsymbol{\Upsilon}^{(g-1)}
\end{array}\right]-\boldsymbol{J}^{-1}\left[\begin{array}{c}
\boldsymbol{F}^{\mathrm{tot}}-\boldsymbol{F}^{\mathrm{d}} \\
\boldsymbol{Z}-\boldsymbol{Z}^{\mathrm{d}} \\
\boldsymbol{\Gamma}_{Z^{\mathrm{d}}, n}
\end{array}\right]
$$

where $\boldsymbol{J}$ is the Jacobian matrix.
As seen in Fig. 4, we loop on frictional contact problem followed by a Newton step that gives a new positionorientation of foot. To increase the speed of the algorithm, the precision of the frictional contact problem is increased with the convergence of the Newton steps.

Using the previous equations, we can easily calculate the reference ankle position and orientation in the control scheme 2 during the contact phase using the following equation:

$$
\boldsymbol{P}_{a}=\boldsymbol{O}_{l}+\boldsymbol{R}^{l} \boldsymbol{P}_{a}
$$

where ${ }^{l} \boldsymbol{P}_{a}$ is the ankle position in the sole frame.

When the foot is not in contact with the floor, ankle positions and orientations are obtained by interpolations of $5^{\text {th }}$ order polynomials between the absolute ankle orientation, position, velocity and acceleration of take-off and landing phase.

\section{Initial conditions}

To solve the previous problem, we set conditions at the first time step. We imposed an initial position $\boldsymbol{O}_{l}^{\text {ini }}$ and yaw orientation $\psi^{\text {ini }}$ of the foot just before the first contact that is used as reference contact position $\boldsymbol{Q}$. Initial roll and pitch orientation are not imposed since it could be discontinuous with the roll and pitch orientation found from desired ZMP position. Initial roll and pitch orientation of the foot are then equal as those obtained from the desired ZMP position. Therefore, tangential reference contact positions are:

$$
\boldsymbol{Q}_{t}=\mathbf{t} \boldsymbol{O}_{l}^{\mathrm{ini}}+\mathbf{t} \boldsymbol{R}^{\mathrm{ini}}\left(\theta, \phi, \psi^{\mathrm{ini}}\right){ }^{l} \boldsymbol{P}_{\alpha}^{\text {free }}
$$

Newton step (19) at the first time step changed accordingly.

\section{MUlTi-OBJECTIVE QUADRATIC PROGRAM CONTROLLER}

From previous offline steps, we obtained reference trajectories for COM, ankle position and orientation. The goal of the QP is to track online those references as well as possible while satisfying robot constraints. We used a taskspace QP formulation with a weighted hierarchy [4] to control the whole body motion. The optimization variables are $\boldsymbol{x}=\left[\begin{array}{ll}\ddot{\boldsymbol{q}}^{T} & \boldsymbol{\lambda}^{T}\end{array}\right]^{T}$ where $\ddot{\boldsymbol{q}}$ is the joint acceleration vector and $\lambda$ is the force intensity along with the linearized friction cone.

We formulated the QP in the following way:

$$
\min _{\boldsymbol{x}}\left(\frac{1}{2} \boldsymbol{x}^{T} \boldsymbol{Q} \boldsymbol{x}+\boldsymbol{c}^{T} \boldsymbol{x}\right)=\min _{\boldsymbol{x}} \sum_{i=1}^{N} \alpha_{i}\left\|E_{i}(\boldsymbol{x})\right\|^{2}+\alpha_{\lambda}\|\lambda\|^{2}
$$

where $N$ is the task number, $E_{i}(\boldsymbol{x})$ is the task errors, $\alpha_{i}$ is the task weight and $\alpha_{\lambda}\|\lambda\|^{2}$ is a damping term to ensure positive definite Hessian matrix.

The constraints are:

1) Joint torque limits

2) Joint position/velocity limits

3) No-sliding contacts: zero acceleration for the contact body $i$ 


\section{4) Collision avoidance}

The tasks are:

1) Ankle: desired ankle trajectories given by the deformation estimator

2) COM: tracking of the desired COM position, velocity and acceleration given by the WPG

3) Torso: fixed torso absolute orientation

4) Posture: fixed posture for the whole upper body (torso, head, and arms)

\section{Results}

In this section, we compare the results obtained with and without our deformation estimator. The advantages to use feet with flexible sole instead of the common feet with ankle flexibility is explained thoroughly in [8].

Fig. 5 shows HRP-4's new feet.

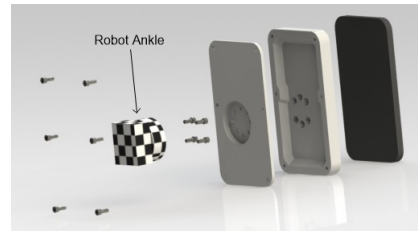

(a)

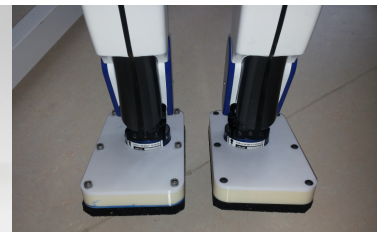

(b)
Fig. 5: (a): new HRP-4's feet; (b): photo of HRP-4's feet with flexible soles

We used two sole materials: flexible foam and an antishock plate. To obtain the Young and Poisson modulus of these materials, we coupled compression test and FEM simulation. Table I details these identification results.

TABLE I: Material identification results

\begin{tabular}{llll}
\hline Material & Guessed type & E (MPa) & $\nu$ \\
\hline Flexible foam & Polyethylene & 0.24 & 0.31 \\
Anti-shock plate & Polyvinyl chloride & 7 & 0.48 \\
\hline
\end{tabular}

Experiments consisted of HRP-4's straightforward walking for 10 footsteps (distance of $0.5 \mathrm{~m}$ ). In this paper, we used ZMP and COM trajectories obtained using the WPG described in section II with $\lambda=0.6$ (a value which allows a heel-toe trajectory and showed instability problem for HRP2's straightforward walking in [8]) and $\epsilon=15$ in (1). These trajectories are shown in Fig. 6a. Fig. 6b shows the ankle trajectories when the foot is not in contact with the floor.

From the inverse pendulum model and the ZMP equation [6], the COM acceleration is an image of the ZMP position in the COM frame (ignoring a multiplication factor). To avoid noisy force measurements due to the impact-shock at the landing phase or drift from acceleration integration, we focused on the analyse of the COM acceleration given by the robot Inertia Measurement Unit (IMU).

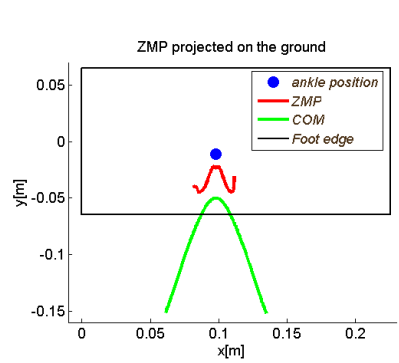

(a)

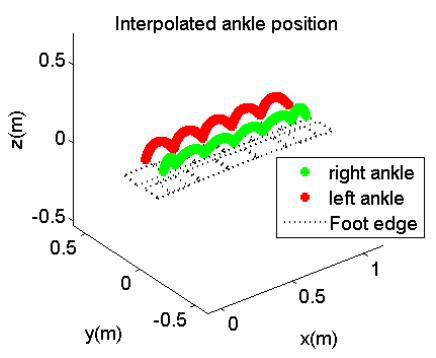

(b)
Fig. 6: (a): ZMP trajectory of the left foot and COM trajectory during a foot step (SSP+DSP); (b): ankle trajectories when the foot is not in contact with the floor

In the video attached to this paper and in Fig. 7, we can clearly see the improvements given by the deformation estimator. Figs. 7a, 7b and 7c shows the COM acceleration for the experiment with the flexible foam. During this experiment, when we did not use the deformation estimator the robot fell down (the problem of the COM acceleration tracking is clearly identifiable around $4 \mathrm{~s}$ ). When we used the deformation estimator the COM acceleration along with $\mathrm{y}$-axis is close to the reference acceleration (see Fig. 7b). On the contrary, the COM acceleration along with $\mathrm{x}$-axis and $\mathrm{z}$-axis are quite different from the reference acceleration (see Figs. 7a and 7c). These tracking errors arise from the use of the HRP-4's native stabilizer that does not take into account the new sole flexibilities, and from some conflicting tasks and constraints of the multi-objective QP controller (see section IV).

We can therefore say that the deformation estimator is a good model of the flexible foam flexibility and enhances the native stabilizer to keep the desired robot attitude during the walking task.

Figs. 7d, 7e and 7f shows the COM acceleration for the experiment with the anti-shock plate. In this case, the robot keep its balances with and without the deformation estimator. Compared to the flexible foam, the sole deformations are very low and the WPG can generate stable ZMP trajectories for not so high deformable sole material. This result is shown in [8]. Nevertheless, the deformation estimator allows a better tracking of the reference trajectories. In Fig. 7e the COM acceleration in $y$-axis is close to the reference with the deformation estimator, but the tracking error for the COM acceleration is bigger when we did not use the deformation estimator than when we used it. As for the walk with the soles made in flexible foam, there is a vertical COM acceleration. The tracking errors of the COM acceleration along with $\mathrm{x} / \mathrm{z}$-axes are also bigger when we did not use the deformation estimator than when we used it (see Figs. $7 d$ and 7f).

Thus, even with low deformation, the effects of the deformation estimator are visible in the reference tracking error. 


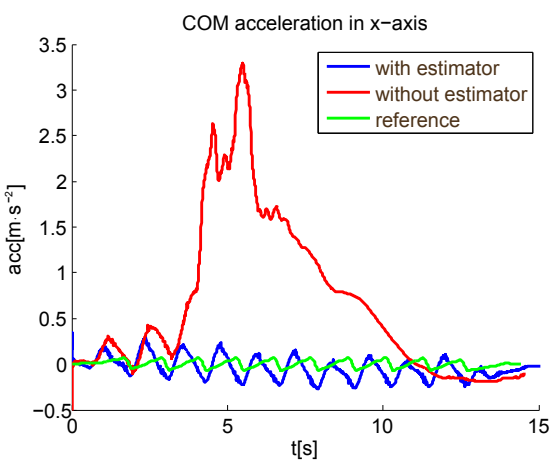

(a)

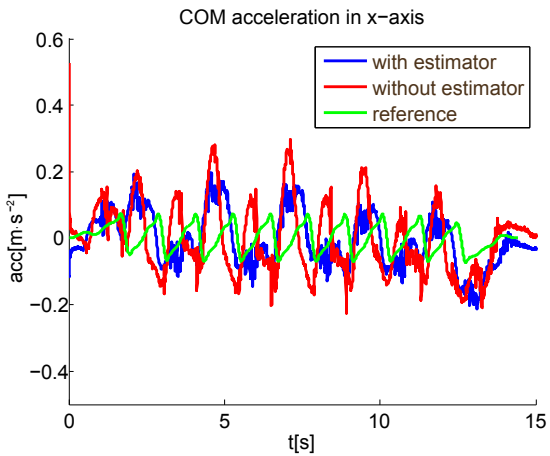

(d)

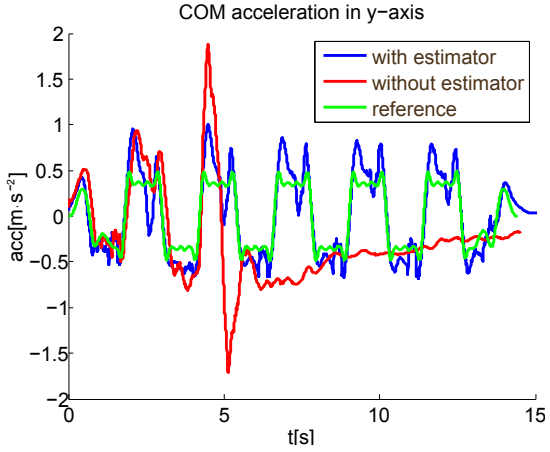

(b)

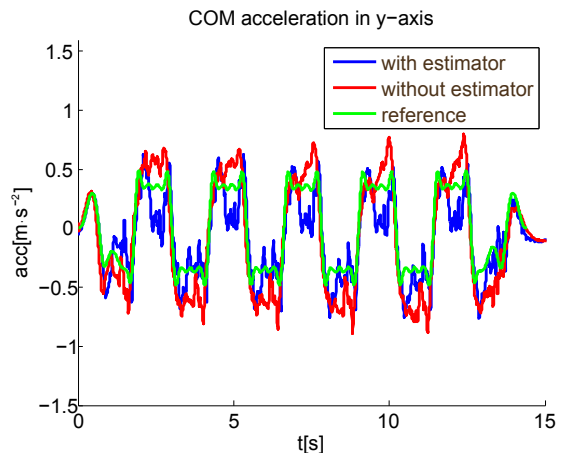

(e)

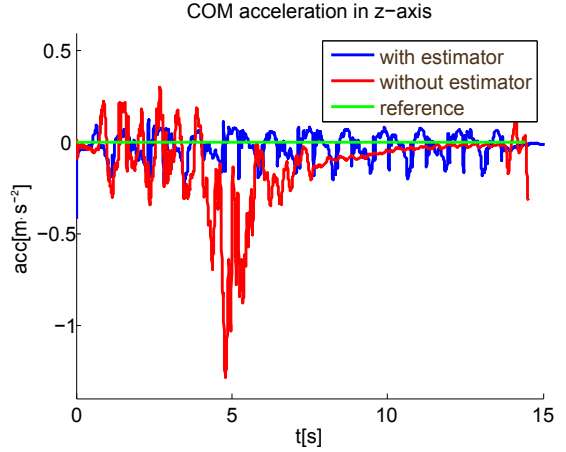

(c)

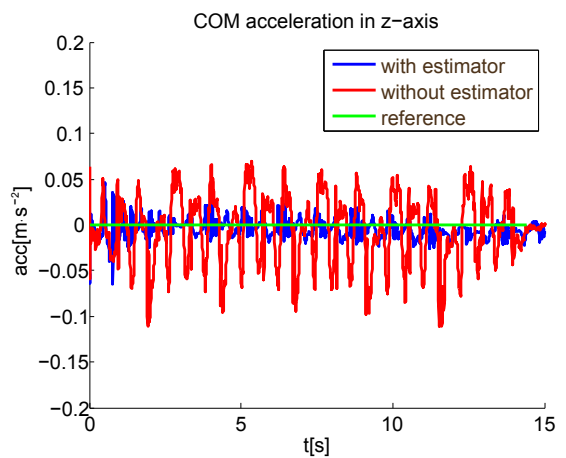

(f)

Fig. 7: $(\mathrm{a}, \mathrm{b}, \mathrm{c})$ Flexible foam and $(\mathrm{d}, \mathrm{e}, \mathrm{f})$ anti-shock plate: measured COM acceleration compared to the reference acceleration along with $\mathrm{x} / \mathrm{y} / \mathrm{z}$ axes with and without the deformation estimator

\section{CONCLUSION}

We developed a WPG based on the minimization of energy consumption that allows having smooth heel-toe human-like ZMP force and trajectory under each foot. This WPG gives the right input to the new deformation estimator developed in this paper. Using this estimator, we take into account the flexibility using a FEM of the sole and a contact model that conforms the mechanical laws.

Experiments showed how the estimator enhances the ZMP stabilization during humanoid walking with flexible soles of different materials. In future work, we will improve the robustness of this approach with a dedicated stabilizer.

\section{REFERENCES}

[1] K. Bouyarmane and A. Kheddar, "Humanoid robot locomotion and manipulation step planning," Advanced Robotics, no. 26, pp. 1099 1126, July/September 2012

[2] K. Hirai, M. Hirose, Y. Haikawa, and T. Takenaka, "The development of honda humanoid robot," in IEEE International Conference on Robotics and Automation, vol. 2. Leuven, Belgium: IEEE, May 1998, pp. 1321-1326.

[3] K. Kaneko, F. Kanehiro, S. Kajita, H. Hirukawa, T. Kawasaki, M. Hirata, K. Akachi, and T. Isozumi, "Humanoid robot hrp-2," in IEEE International Conference on Robotics and Automation, New Orleans, USA, April 2004, pp. 1083-1090.

[4] J. Vaillant, A. Kheddar, H. Audren, F. Keith, S. Brossette, A. Escande, K. Bouyarmane, K. Kaneko, M. Morisawa, P. Gergondet, E. Yoshida, S. Kajita, and F. Kanehiro, "Multi-contact vertical ladder climbing with an hrp-2 humanoid," Autonomous Robots, vol. 40, no. 3, pp. 561-580, 2016
[5] J. Yamaguchi and A. Takanishi, "Multisensor foot mechanism with shock absorbing material for dynamic biped walking adapting to unknown uneven surfaces," in IEEE International Conference on Multisensor Fusion and Integration for Intelligent systems, Washington DC, USA, December 1996, pp. 233-240.

[6] S. Kajita and B. Espiau, Handbook of Robotics. Springer-Verlag, 2008, ch. Legged Robots, pp. 361-389.

[7] H.-K. Shin and B. K. Kim, "Energy-efficient gait planning and control for biped robots utilizing the allowable zmp region," Robotics, IEEE Transactions on, vol. 30, no. 4, pp. 986-993, August 2014.

[8] A. Pajon, G. De Magistris, S. Miossec, K. Kaneko, and A. Kheddar "A humanoid walking pattern generator for sole design optimization,' in International Conference on Advanced Robotics (ICAR), Istanbul, Turkey, 2015.

[9] M. Bonnet, A. Frangi, and C. Rey, The finite element method in solid mechanics. McGraw Hill Education, 2014. [Online]. Available: https://hal.archives-ouvertes.fr/hal-01083772

[10] M. Bro-Nielsen and S. Cotin, "Real-time volumetric deformable models for surgery simulation using finite elements and condensation,' Computer Graphics Forum (Eurographics'96), vol. 15, p. 17, 1996.

[11] J.-J. Moreau and M. Jean, "Numerical treatment of contact and friction: The contact dynamics method," Engineering Systems Design and Analysis, vol. 4, no. 1, pp. 201-208, 1996.

[12] M. Jean, "Numerical methods for three dimensional dynamical problems," in Conference in Contact Mechanics, Southampton, 1993, p. 71.

[13] P. Alart and A. Curnier, "Time-stepping for threedimensal rigid body dynamics," Computer Methods in Applied Mechanics and Engineering, vol. 92, no. 3, pp. 353-375, November 1991.

[14] C. Duriez, F. Dubois, A. Kheddar, and C. Andriot, "Realistic haptic rendering of interacting deformable objects in virtual environments," IEEE Transactions on Visualization and Computer Graphics, vol. 12 , no. 1 , pp. $36-47,2006$

[15] F. Jourdan, P. Alart, and M. Jean, "A gauss-seidel like algorithm to solve frictional contact problems," Computer Methods in Applied Mechanics and Engineering, pp. 33-47, 1998. 